\title{
Condensation Transitions in Nonequilibrium Systems
}

\author{
M.R. Evans
}

\begin{abstract}
Systems driven out of equilibrium can often exhibit behaviour not seen in systems in thermal equilibrium- for example phase transitions in one-dimensional systems. In this talk I will review several 'condensation' transitions that occur when a conserved quantity is driven through the system. Although the condensation is spatial, i.e. a finite fraction of the conserved quantity condenses into a small spatial region, useful comparison can be made with usual Bose-Einstein condensation. Amongst some one-dimensional examples I will discuss the 'Bus Route Model' where the condensation corresponds to the clustering together of buses moving along a bus-route.
\end{abstract}

\section{Introduction}

Broadly speaking, one can consider two types of nonequilibrium systems: those relaxing towards thermal equilibrium and those held far from thermal equilibrium e.g. by the system being driven by some external field. In the latter case the steady state of the system will not be described by usual Gibbs-Boltzmann statistical weights rather it will be a nonequilibrium steady state. A natural way to construct a nonequilibrium steady state is to drive the system by forcing a current of some conserved quantity, for example energy or mass, through the system. Such systems are known as driven diffusive systems (DDS) [2].

In recent years the possibility of phase transitions and phase separation in one-dimensional nonequilibrium systems has been explored and some examples are by now well studied. To appreciate the significance one should recall the general dictum that in one-dimensional equilibrium systems phase ordering and phase transitions do not occur (except in the limit of zero-temperature, or with long range interaction) [3].

Let us briefly review work on one-dimensional phase transitions in driven systems. A very simple one-dimensional driven diffusive system is the asymmetric simple exclusion process (ASEP). Here particles hop in a preferred direction on a one-dimensional lattice with hard-core exclusion (at most one particle can be at any given site). Indicating the presence of a particle by a 1 and an empty site (hole) by 0 the dynamics comprises the following exchanges at nearest neighbour sites

$$
\begin{aligned}
& 10 \rightarrow 01 \\
& 01 \rightarrow 10
\end{aligned} \begin{aligned}
& \text { with rate } \\
& 1 \\
&
\end{aligned}
$$


The open system was studied by Krug[4] and boundary induced phase transitions shown to be possible. Specifically one considers a lattice of $N$ sites where at the left boundary site (site 1) a particle is introduced with rate $\alpha$ if that site is empty, and at the right boundary site ( site $N$ ) any particle present is removed with rate $\beta$. Thus the dynamical processes at the boundaries are

$$
\begin{aligned}
& \text { at site } 1 \quad 0 \rightarrow 1 \text { with rate } \alpha \\
& \text { at site } N 1 \rightarrow 0 \text { with rate } \beta \text {. }
\end{aligned}
$$

These boundary conditions force a steady state current of particles $J$ through the system. Phase transitions occur when $\lim _{N \rightarrow \infty} J$ exhibits non-analyticities. The steady state of this system was solved exactly for the totally asymmetric case $[5,6]$ and more recently for the general $q$ case $[7,8]$. When $q<1$ the phase diagram comprises three phases: a high-density phase where the current is controlled by a low exit rate $\beta$ - one can think of this is queue of cars at a traffic light that doesn't let many cars through; a low-density phase where the current controlled by a low injection rate $\alpha$-think of this as a traffic light that does not let many cars onto an open road; a maximal-current phase where both $\alpha, \beta$ are high $(\alpha, \beta>(1-q) / 2)$ and the current is $J=(1-q) / 4$. Note that since increasing $\alpha$ and $\beta$ doesn't increase the current, the current is saturated. In the maximal current phase generic longrange correlations exist, an example being the decay of particle density from the left boundary to the bulk value $1 / 2$ which is a power law $\sim 1 / x^{1 / 2}$ where $x$ is distance from the left boundary.

On the line $\alpha=\beta<(1-q) / 2$ which separates the high and low density phases one finds coexistence between a region of low density in the left part of lattice and a region of high density on the right separated by a 'shock' where the density changes sharply over a microscopic distance.

Perodic systems (i.e. a ring of sites) can also exhibit phase separation when inhomogeneities or defects are introduced. A very simple example is to introduce into the asymmetric exclusion process a 'slow bond' through which particles hop with a reduced rate. Then in the steady state one can obtain phase separation between a region of high density behind the slow bond and a region of low density in front of the slow bond. Moving defects (i.e. particles with dynamics different from that of the others) have also been considered and exact solutions obtained $[11,12,13]$. One can think of a slow agricultural vehicle on a country road with a large queue of cars behind it and open road in front of it.

A further question is whether systems related to the hopping particle models described so far, but without inhomogeneities, can exhibit phase ordering. A very simple model was introduced in [14] comprising three species of conserved particles, amongst which all possible exchanges are allowed. However a key feature is that the dynamics has a cyclic symmetry i.e. $A$ particles move preferentially to the left of $B$ particles which move preferentially to the left of $C$ particles which in turn move preferentially to the left of $A$ particles. The model exhibits strong phase 
separation into pure domains of $A B C$. Similar strong phase separation occurs in other related models [15].

A final class of transitions in one-dimensional hopping particle models is that involving spatial condensation, whereby a finite fraction of the particles condenses onto the same site. Examples include the appearance of a large aggregate in models of aggregation and fragmentation[16] and the emergence of a single flock in dynamical models of flocking [17]. We will analyze a simple example of a condensation transition which occurs in the zero-range process which we now define.

\section{The zero-range process}

The zero-range process was introduced by Spitzer [9] and recent applications and developments have been reviewed in [10]. We consider a one-dimensional lattice of $M$ sites with sites labeled $\mu=1 \ldots M$ and periodic boundary conditions (more generally one can consider the zero-range process on a lattice of arbitrary dimension). Each site can hold an integer number of indistinguishable particles. The configuration of the system is specified by the occupation numbers $n_{\mu}$ of each site $\mu$. The total number of particles is denoted by $L$ and is conserved under the dynamics. The dynamics of the system is given by the rates at which a particle leaves a site $\mu$ (one can think of it as the topmost particle - see Figure 1a) and moves to the left nearest neighbour site $\mu-1$. The hopping rates $u(n)$ are a function of $n$ the number of particles at the site of departure. Some particular cases are: if $u(n)=n$ then the dynamics of each particle is independent of the others; if $u(n)=$ const for $n>0$ then the rate at which a particle leaves a site is unaffected by the number of particles at the site (as long as it is greater than zero).

The important attribute of the zero-range process is that it has a steady state described by a product measure. By this it is meant that the steady state probability $P\left(\left\{n_{\mu}\right\}\right)$ of finding the system in configuration $\left\{n_{1}, n_{2} \ldots n_{M}\right\}$ is given by a product of factors $f\left(n_{\mu}\right)$

$$
P\left(\left\{n_{\mu}\right\}\right)=\frac{1}{Z(M, L)} \prod_{\mu=1}^{M} f\left(n_{\mu}\right) .
$$

Here the normalization $Z(M, L)$ is introduced so that the sum of the probabilities for all configurations, with the correct number of particles $L$, is one.

In the basic model described above, $f(n)$ is given by

$$
\begin{aligned}
f(n) & =\prod_{m=1}^{n} \frac{1}{u(m)} \text { for } n \geq 1 \\
& =1 \text { for } n=0
\end{aligned}
$$

To prove $(3,4)$ one simply considers the stationarity condition on the probability of a configuration (probability current out of the configuration due to hops 
is equal to probability current into the configuration due to hops):

$$
\begin{aligned}
& \sum_{\mu} \theta\left(n_{\mu}\right) u\left(n_{\mu}\right) P\left(n_{1} \ldots n_{\mu} \ldots n_{L}\right)= \\
& \sum_{\mu} \theta\left(n_{\mu}\right) u\left(n_{\mu+1}+1\right) P\left(n_{1} \ldots n_{\mu}-1, n_{\mu+1}+1 \ldots n_{L}\right) .
\end{aligned}
$$

The Heaviside function $\theta\left(n_{\mu}\right)$ highlights that it is the sites with $n>1$ that allow exit from the configuration (lhs of (5)) but also allow entry to the configuration (rhs of (5)). Equating the terms $\mu$ on both sides of (5) and cancelling common factors assuming (3), results in

$$
u\left(n_{\mu}\right) f\left(n_{\mu-1}\right) f\left(n_{\mu}\right)=u\left(n_{\mu+1}+1\right) f\left(n_{\mu}-1\right) f\left(n_{\mu+1}+1\right)
$$

This equality can be recast as

$$
u\left(n_{\mu}\right) \frac{f\left(n_{\mu}\right)}{f\left(n_{\mu}-1\right)}=u\left(n_{\mu+1}+1\right) \frac{f\left(n_{\mu+1}+1\right)}{f\left(n_{\mu+1}\right)}=\mathrm{constant}
$$

Setting the constant equal to unity implies

$$
f\left(n_{\mu}\right)=\frac{f\left(n_{\mu}-1\right)}{u\left(n_{\mu}\right)}
$$

and iterating (8) leads to (4).

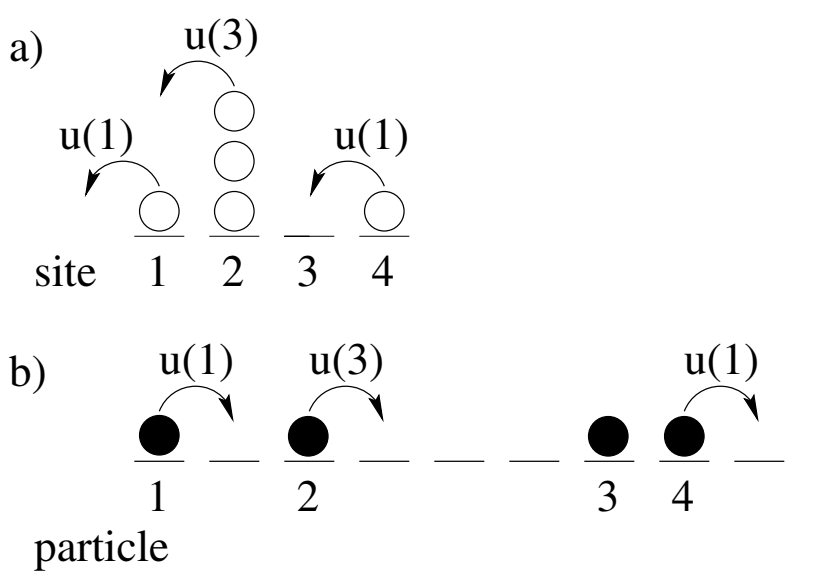

Figure 1: Equivalence of zero range process and asymmetric exclusion process.

We can easily generalize to consider an inhomogeneous system by which we mean the hopping rates are site dependent: the hopping rate out of site $\mu$ when it 
contains $n_{\mu}$ particles is $u_{\mu}\left(n_{\mu}\right)$. It is easy to check that the steady state is simply modified to

$$
P\left(\left\{n_{\mu}\right\}\right)=\frac{1}{Z(M, L)} \prod_{\mu=1}^{L} f_{\mu}\left(n_{\mu}\right)
$$

where $f_{\mu}$ are given by

$$
\begin{aligned}
f_{\mu}(n) & =\prod_{m=1}^{n} \frac{1}{u_{\mu}(m)} \quad \text { for } \quad n \geq 1 \\
& =1 \quad \text { for } \quad n=0
\end{aligned}
$$

The proof is identical to that for the homogeneous case, with the replacement of $u\left(n_{\mu}\right)$ by $u_{\mu}\left(n_{\mu}\right)$

There exists an exact mapping from a zero-range process to an asymmetric exclusion process. This is illustrated in Figure 1. The idea is to consider the particles of the zero-range process as the holes (empty sites) of the exclusion process. Then the sites of the zero-range process become the moving particles of the exclusion process. Note that in the exclusion process we have $M$ particles hopping on a lattice of $M+L$ sites. A hopping rate in the zero range process $u(m)$ which is dependent on $m$ corresponds to a hopping rate in the exclusion process which depends on the gap to the particle in front. So the particles can feel each other's presence and one can have a long-range interaction.

\section{Condensation Transitions}

We now proceed to analyze the steady states of form (9) and the condensation transition that may occur. The important quantity to consider is the normalization $Z(M, L)$ as it plays the role of the partition sum. The normalization is defined through the condition

$$
Z(M, L)=\sum_{n_{1}, n_{2} \ldots n_{M}} \delta\left(\sum_{\mu} n_{\mu}-L\right) \prod_{\mu=1}^{M} f_{\mu}\left(n_{\mu}\right)
$$

where the $\delta$ function enforces the constraint of $L$ particles. The normalization may be considered as the analogue of a canonical partition function of a thermodynamic system.

We define the 'speed' $v$ as the average hopping rate out of a site

$$
\begin{aligned}
v & =\frac{1}{Z(M, L)} \sum_{n_{1}, n_{2} \ldots n_{M}} \delta\left(\sum_{\mu} n_{\mu}-L\right) u\left(n_{1}\right) \prod_{\mu=1}^{M} f_{\mu}\left(n_{\mu}\right) \\
& =\frac{Z(M, L-1)}{Z(M, L)}
\end{aligned}
$$


where we have used $(9,10)$. Note that $(12)$ tells us that the speed is independent of site and thus may be considered a conserved quantity in the steady state of the system. In the totally asymmetric system considered in Section 2 the speed is equal to the current of particles flowing between neighbouring sites. The speed is a ratio of partition functions of different system sizes (12) and corresponds to a fugacity.

We now use the integral representation of the delta function to write the partition function as

$$
Z(M, L)=\oint \frac{d z}{2 \pi i} z^{-(L+1)} \prod_{\mu=1}^{M} F_{\mu}(z),
$$

where

$$
F_{\mu}(z)=\sum_{m=0}^{\infty} z^{m} f_{\mu}(m) .
$$

For large $M, L(13)$ is dominated by the saddle point of the integral and the value of $z$ at the saddle point is the fugacity. The equation for the saddle point reduces to

$$
\frac{L}{M}=\frac{z}{M} \sum_{\mu=1}^{M} \frac{\partial}{\partial z} \ln F_{\mu}(z)
$$

which, defining $\phi=L / M$, can be written as

$$
\phi=\frac{z}{M} \sum_{\mu=1}^{M} \frac{F_{\mu}^{\prime}(z)}{F_{\mu}(z)} .
$$

In the thermodynamic limit,

$$
M \rightarrow \infty \text { with } L=\phi M
$$

where the density $\phi$ is held fixed, the question is whether a valid saddle point value of $\mathrm{z}$ can be found from (16). We expect that for low $\phi$ the saddle point is valid but, as we shall discuss, there exists a maximum value of $z$ and if at this maximum value the rhs of (16) is finite, then for large $\phi(16)$ cannot be satisfied. We now consider how condensation may occur in the inhomogeneous and the homogeneous case.

\subsection{Inhomogeneous case}

To give an idea of how a condensation transition may occur we consider the case $u_{\mu}(m)=u_{\mu}$ for $m>0$ i.e. the hopping rate does not depend on the number of particles at a site. $f_{\mu}$ is given by

$$
f_{\mu}(n)=\left(\frac{1}{u_{\mu}}\right)^{n_{\mu}}
$$


and the probability of occupancies $\left\{n_{1}, n_{2}, \ldots, n_{M}\right\}$ is

$$
P\left(\left\{n_{1}, n_{2}, \ldots, n_{M}\right\}\right)=\frac{1}{Z(M, L)} \prod_{\mu=1}^{M}\left(\frac{1}{u_{\mu}}\right)^{n_{\mu}} .
$$

The mapping to an ideal Bose gas is evident: the $L$ particles of the zero-range process are viewed as Bosons which may reside in $M$ states with energies $E_{\mu}$ determined by the site hopping rates: $\exp \left(-\beta E_{\mu}\right)=1 / u_{\mu}$. Thus the ground state corresponds to the site with the lowest hopping rate. The normalization $Z(M, L)$ is equivalent to the canonical partition function of the Bose gas. We can sum the geometric series (14) to obtain $F_{\mu}$ and $F_{\mu}^{\prime}$ then taking the large $M$ limit allows the sum over $\mu$ to be written as an integral

$$
\phi=\int_{u_{\min }}^{\infty} d u \mathcal{P}(u) \frac{z}{u-z}
$$

where $\mathcal{P}(u)$ is the probability distribution of site hopping rates with $u_{\text {min }}$ the lowest possible site hopping rate. Interpreting $\mathcal{P}(u)$ as a density of states, equation (20) corresponds to the condition that in the grand canonical ensemble of an ideal Bose gas the number of Bosons per state is $\phi$. The theory of Bose condensation tells us that when certain conditions on the density of low energy states pertain we can have a condensation transition. Then (16) can no longer be satisfied and we have a condensation of particles into the ground state, which is here the site with the slowest hopping rate.

A very simple example is to have just one 'slow site' i.e. $u_{1}=p$ while the other $M-1$ sites have hopping rates $u_{\mu}=1$ when $\mu>1$. Using the mapping to an exclusion process, this corresponds one slow particle i.e. agricultural vehicle example described earlier. One can show [11] that for a high density of particles in the zero range process (low density of particles in the corresponding asymmetric exclusion process) we have a condensate since site 1 contains a finite fraction of the particles. In the low density phase the particles are evenly spread between all sites.

\subsection{Homogeneous case}

We now consider the homogeneous zero-range process where the hopping rates $u(n)$ are site independent. Then (14) is independent of $\mu$ and reads

$$
F(z)=\sum_{n=0}^{\infty} \prod_{m=1}^{n}\left[\frac{z}{u(m)}\right]
$$

The fugacity $z$ must be chosen so that $F$ converges or else we could not have performed (14). Therefore $z$ is restricted to $z \leq \beta$ where we define $\beta$ to be the radius of convergence of $F(z)$. From (21) we see that $\beta$ is the limiting value of the 
$u(m)$ i.e. the limiting value of the hopping rate out of a site for a large number of particles at a site. We interpret (16) as giving a relation between the density of holes (number of holes per site) and the fugacity $z$. The saddle point condition (16) becomes

$$
\phi=\frac{z F^{\prime}(z)}{F(z)}
$$

Given that the rhs of (22) is a monotonically increasing function of $z$ we deduce that density of particle increases with fugacity. However if at $z=\beta$, the maximum allowed value of $z$, the rhs of (22) is still finite then one can no longer solve for the density and one must have a condensation transition. Physically, the condensation would correspond to a spontaneous symmetry breaking where one of the sites is spontaneously selected to hold a finite fraction of the particles.

Thus, for condensation to occur (i.e. when $\phi$ is large enough for (22) not to have a solution for the allowed values of $z$ ) we require

$$
\lim _{z \rightarrow \beta} \frac{F^{\prime}(z)}{F(z)}<\infty .
$$

We now assume that $u(n)$ decreases uniformly to $\beta$ in the large $n$ limit as

$$
u(n)=\beta(1+\zeta(n))
$$

where $\zeta(n)$ is a monotonically decreasing function. Analysis of the series

$$
\begin{aligned}
& F(\beta)=\sum_{n=0}^{\infty} \exp \left\{-\sum_{m=1}^{n} \ln [1+\zeta(m)]\right\} \\
& F^{\prime}(\beta)=\sum_{n=0}^{\infty} n \exp \left\{-\sum_{m=1}^{n} \ln [1+\zeta(m)]\right\}
\end{aligned}
$$

reveals that the condition for condensation is simply that $F^{\prime}(\beta)$ is finite and this occurs if $u(n)$ decays to $\beta$ more slowly than $\beta(1+2 / n)$. (This is easiest to see by expanding $\ln [1+\zeta]$ and approximating the sum over $m$ by an integral in (25).)

It is interesting to translate this result into the language of the exclusion process. In this context we can have condensation if the hop rate of a particle into a gap of size $n$ decays as $\beta(1+2 / n)$ therefore there is an effective long range interaction.

\subsection{Bus route model}

As an example of this let us consider the 'bus route model' [18]. The model is defined on a $1 d$ lattice. Each site (bus-stop) is either empty, contains a bus (a conserved particle) or contains a passenger (non-conserved quantity). The dynamical processes are that passengers arrives at an empty site with rate $\lambda$; a bus moves 
forward to the next stop with rate 1 if that stop is empty; if the next stop contains passengers the bus moves forward with rate $\beta$ and removes the passengers.

The model thus defined has not been solved but simulations reveal two regimes. At high bus density the gaps between buses are evenly distributed. However at low bus density there is a condensed regime where the lead bus has a large gap to the next bus in front of it with bus-stops full of passengers in between. The other buses have small gaps between them. Thus the buses form a jam of buses and after a long delay all arrive at a bus-stop at once.

The bus route model can be related to the zero-range process by a mean-field approximation in which we integrate out the non-conserved quantity (passengers). The idea is that a bus-stop, next to bus 1 say, will last have been visited by a bus (bus 2) a mean time ago of $n / v$ where $n$ is the distance between bus 2 and bus 1 and $v$ is the steady state speed. Therefore the mean-field probability that the site next to bus 1 is not occupied by a passenger is $\exp (-\lambda n / v)$. From this probability an effective hopping rate for a bus into a gap of size $n$ is obtained by averaging the two possible hop rates $1, \beta$ :

$$
u(n)=\beta+(1-\beta) \exp (-\lambda n / v) .
$$

We can now see that this mean-field approximation to the bus-route model is equivalent to a homogeneous zero-range process discussed earlier.

Since $u(n)$ decays exponentially the condition for a strict phase transition in the thermodynamic limit is not met. However on any finite system for $\lambda$ sufficiently small, an apparent condensation will be seen. In the bus route problem this corresponds to the universally irritating situation of all the buses on the route arriving at once.

\section{Conclusion}

We have shown how the zero range process exhibits two kinds of condensation transition. One is due to having an inhomogeneous system i.e. we get condensation of particles onto the site with the slowest hopping rate. Although the condensation is spatial the mechanism is equivalent to Bose condensation in an Ideal Bose Gas.

The other type of condensation occurs on a homogeneous systems and involves the spontaneous selection of a site onto which a finite fraction of the particles condense. Recently this condensation mechanism has been used to understand the existence or non-existence of phase separation in a general class of one dimensional driven systems $[19,20]$.

\section{References}

[1] S. Katz, J.L. Lebowitz and H. Spohn, Phys. Rev. B 28, 1655 (1983); J. Stat. Phys 34497 (1984). 
[2] B. Schmittmann and R.K.P. Zia, Statistical Mechanics of Driven Diffusive Systems vol. 17 of Domb and Lebowitz series, Academic Press, U.K. (1995).

[3] L.D. Landau and E.M. Lifshitz, 1980, Statistical Physics I (Pergamon Press, New York).

[4] J. Krug, Phys. Rev. Lett. 67, 1882 (1991).

[5] B. Derrida, M.R. Evans, V. Hakim and V. Pasquier, J. Phys. A 26, 1493 (1993).

[6] G. Schütz and E. Domany, J. Stat. Phys. 72, 277 (1993).

[7] T. Sasamoto, J. Phys. A 32, 7109 (1999).

[8] R. A. Blythe, M. R. Evans, F. Colaiori and F. H. L. Essler, J. Phys. A 33, $2313(2000)$.

[9] F. Spitzer, Advances in Math. 5, 246 (1970).

[10] M. R. Evans, Brazilian Journal of Physics 30, 42 (2000).

[11] M. R. Evans, Europhys. Lett. 36, 13 (1996).

[12] J. Krug and P. A. Ferrari, J. Phys. A 29, L465 (1996).

[13] K. Mallick, J. Phys. A 29, 5375 (1996).

[14] M. R. Evans, Y. Kafri, H. M. Koduvely and D. Mukamel, Phys. Rev. Lett. 80, 425 (1998); Phys. Rev. E 582764 (1998).

[15] R. Lahiri, M. Barma and S. Ramaswamy, Phys. Rev. Lett. 79, 1150 (1997); Phys. Rev. E 61, 1648 (2000).

[16] S. N. Majumdar, S. Krishnamurthy, M. Barma, Phys. Rev. Lett. 81, 3691 (1998); Phys. Rev. E 61, 6337 (2000).

[17] O. J. O'Loan and M. R. Evans, J. Phys. A 32, L99 (1999).

[18] O. J. O'Loan, M.R.Evans and M.E.Cates, Phys. Rev. E. 58, 1404 (1998).

[19] Y. Kafri, E. Levine, D. Mukamel, G. M. Schütz, and J. Torok, Phys. Rev. Lett. 89, 035702 (2002).

[20] Y. Kafri, E. Levine, D. Mukamel and J. Torok, J. Phys. A. 35, L459 (2002).

M.R. Evans

School of Physics

The University of Edinburgh

Mayfield Road

Edinburgh EH9 3JZ

U.K. 